\title{
The intelligent mechatronic system for open pit mining to increase the operation life of equipment
}

\author{
Irina Semykina ${ }^{1, *}$, Valeriy Zavyalov $^{1,2}$, and Veniamin Kashirskikh ${ }^{1}$ \\ ${ }^{1}$ T.F. Gorbachev Kuzbass State Technical University, Department of Electric Drive and Automation, \\ 650000, 28 Vesennyaya Street, Kemerovo, Russia \\ ${ }^{2}$ National Research Tomsk Polytechnic University, Department of Electric Drive and Automation, \\ 634050, 30 Lenin Avenue, Tomsk, Russia
}

\begin{abstract}
This article proposes the intelligent mechatronic system that is based on the controlled induction motor drives. This system is effective to increase the technical operation life of mining machines for open pit mining. It allows control of the dynamic state of the mining machine mechanical components, thus the dynamic loads in the transmission are reduced and the accumulated intensity of fatigue wear becomes smaller. In the beginning, the article describes the concept of such system. The central parts demonstrate the control algorithms of the spring forces in the transmission and observe the estimation of immeasurable mechanical values needed for the mechatronic system operation. In the end of the article, the authors confirm the effectiveness of the proposed solutions experimentally using the data from the acting electric rope shovels.
\end{abstract}

\section{Introduction}

In the open pit mining technology, the mining machines take a central place, and among them the mining excavators are widely distributed. [1]. Despite of the worldwide annual unit production of electric excavators is reducing year by year and the worldwide annual unit production of hydraulic excavators is increasing [2], in Russia not the hydraulic but the electric excavators are most common [3] because of the features of the climate and the production organization. Obviously, the reliability of electric excavators directly affects the mining efficiency, and therefore reliability is the focus of the equipment producers for excavators [4].

By design, any electric excavator is a robotic system with electric drives that perform the operation of movement, rotation and digging [5]. The key feature of this robotic system is the load on the effector that can stochastically vary over a large range until the full locking of the effector when it contacts with solids during the operation of digging. In addition, there is the elastic compliance in the transmission. Together with the variable load the elastic compliance contribute to continuous transient processes in the mechanical

\footnotetext{
* Corresponding author: siyu.eav@kuzstu.ru
} 
elements in transmission [6-14]. As the consequence and one of the main causes of the excavator transmission breakdowns there is accumulation of fatigue damage in them.

The service life of the mining machines for open pit mining can be increased without any changes in the design of mechanisms by using intelligent tools [15]. Such tools may be the introduction of additional measurements or using the controlled induction motor drives, which, for example, control the spring forces in the transmission. The authors believe the latter is most effective, because it is a comprehensive tool. In this case, electric drive can provide a given performance of the excavator by maintaining the mechanical load on the transmission elements in the permitted range, and at the same time it can diminish the accumulation intensity of fatigue damage by control of the dynamic loads, so controlled electric drives can really reduce the likelihood of breakdowns. Noteworthy is that the repair of mechanical transmission breakdowns is the most time consuming [11].

The development of such systems is especially important for Russia, where the average equipment wear rate of excavator fleet is very high. For example, in the largest open-pit coalmines in Kuzbass the wear exceeds $90 \%$ of service life and exceeds $65 \%$ on operating time.

\section{Structure development}

Among the excavator operations the most difficult is the operation of digging, in which two main drives move the bucket through the rock. In the simplest case, the operator controls the movement manually and there is no coordinate control of main drives. Thus, the characteristics of the motors and the properties of the destructible rock both determine the torque and the speed in the transmission elements. There is a strong possibility of motor overload and there are always the significant dynamic loads in transmission.

To avoid this effect, some researchers suggest the more complex control systems where the two main drives of the excavator are working interconnected. For example, the author of [10] proposes the interconnected control system of the drag drive and the hoist drive of the dragline to minimize the energy consumption. Unfortunately, [10] does not regard the improving of the excavator dynamic as a problem.

Another direction of the control systems improving is the individual optimization of one of the main drives in terms of operation life criteria without their collaboration. The most common optimization methods are the identification of the relationship between the parameters of the drives, which provides the best conditions of oscillation damping in transmission. For the transmissions with the elastic compliance there are also the optimization methods based on the subordinate control system with the introduction of additional feedbacks on the spring forces and the speed of the effector. One more optimization method is using of polynomial equations to reduce the rate of response of the control loops. All these methods are united by passive reduction of the dynamic loads.

The most promising control systems of the excavator electric drives are the system with the active limitation of the elastic oscillations where depending on the transmission state the motor forms the electromagnetic torque to suppression of the elastic oscillations. For example, the authors of [12] develop the principles of the control systems for the active limitation of the spring forces oscillations.

So, generalizing the best practices of the control systems of the excavators, the criteria of the intelligent mechatronic systems have been formulated. The system should:

1. Use the perfect type of electric drive.

2. Control the spring forces. That means the system will be able to limit the load on the transmission elements caused by whether the resistance forces of rock destruction or the acceleration and the deceleration and it will be able to reduce the operator influence on the formation of the dynamic loads in the transmission. 
3. Provide the required performance. This means the system must be able to control the bucket speed.

The implementation of these criteria is the basis of the concept of intelligent mechatronic systems, which is shown at Fig. 1 for rope shovel. The diagram denotes: superscript ${ }^{*}$ means the reference value; $\omega_{h}$ - the speed of the dipper handle about the saddle block; $v_{c}$ - the crowd speed; subscripts ${ }_{c}$ and ${ }_{h}$ are related to the crowd drive or the hoist drive values, respectively; $F$ - the spring force; $T$ - the motor torque. In general, the proposed system is quite complex. So, authors have held the decomposition on its mathematical description and for each item the local control tasks are solved.

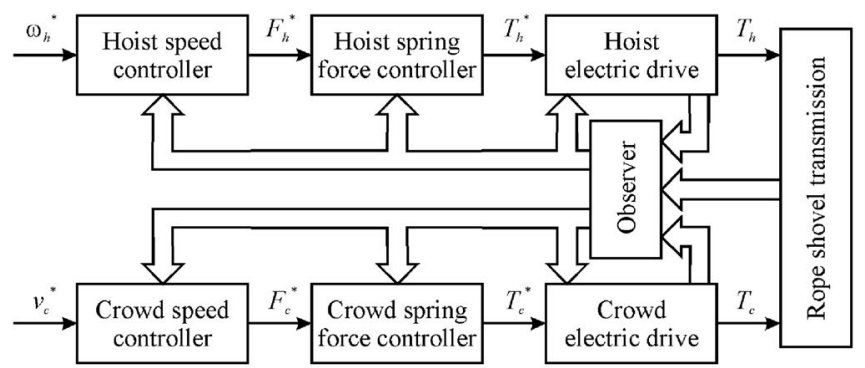

Fig. 1. Block diagram of the proposed intelligent mechatronic systems.

\section{Mathematical model}

From the reliability point of view, the most important for the considered robotic system is the mathematical description of the excavator transmission participated in the operation of digging, because it is a nonlinear system with internal interconnection and variable parameters. To obtain this mathematical description the authors propose to use the model of an interconnected multimass system with elastic coupling. Thus, the mathematical description is based on the transmission kinematics by using the Lagrange equations.

Let us look at the mathematical description of the rope shovel as the multimass system, obtained for the transmission kinematics that is shown in Fig. 2.

a)

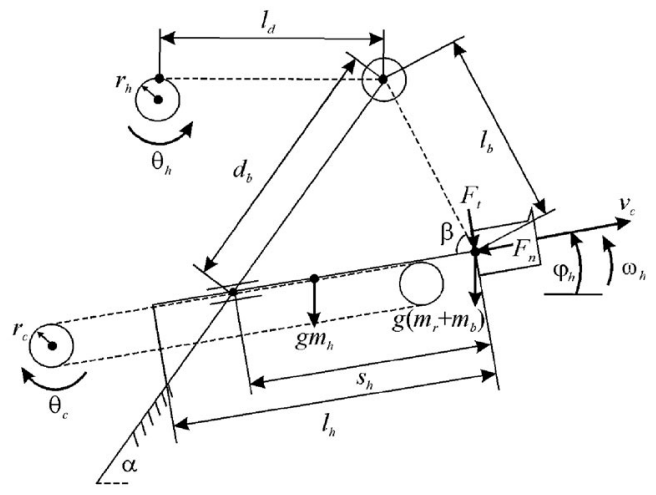

b)

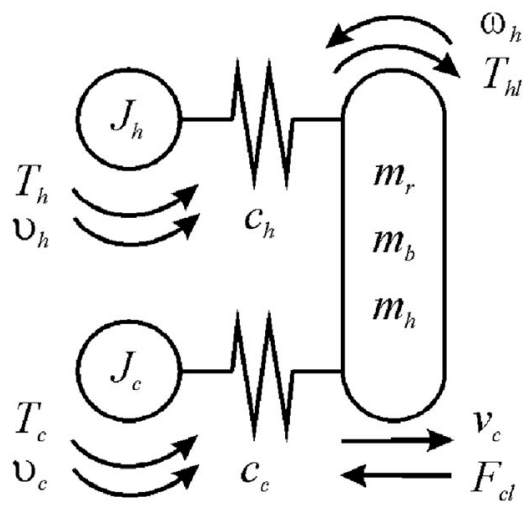

Fig. 2. Rope shovel schemes: a) the transmission kinematics, b) the model of the multimass system.

The schemes denote: $\alpha$ - the boom angle to the horizon; $\beta$ - the hoist cable angle to the dipper handle; $l_{h}$ - the dipper handle length; $d_{b}$ - the boom length from the saddle block to the head block; $l_{d}$ - the hoist cable length from the hoist drum to the boom point sheaves; $l_{b}$ - the hoist cable length from the boom point sheaves to the bucket; $s_{h}$ - the dipper handle 
stroke; $\varphi_{h}$ - the dipper handle angle to the horizon; $F_{t}, F_{n}$ - the tangential and the normal resistance forces of rock destruction; $m_{r}, m_{b}, m_{h}$ - the mass of the rock, the bucket and the dipper handle; $g$ - gravitational acceleration; $F_{l c}$ - the crowd drive load force; $T_{l h}$ - the hoist drive load torque; subscripts ${ }_{c}$ and ${ }_{h}$ relate to the value of the crowd drive or the hoist drive respectively; $r$ - the drum radius; $\theta$ - the drum angle position; $v$ - the drum speed; $J$ - the total specific inertia including the motor, the torque-limiting clutch, the brake, the gear box and the drum; $c$ - the rope specific rigidity rate. A feature of the model is that $m_{r}$ increases during digging.

The mathematical description is the following system of differential equations:

$$
\left\{\begin{array}{l}
\frac{d \theta_{c}}{d t}=v_{c} ; \frac{d \theta_{h}}{d t}=v_{h} ; \frac{d s_{h}}{d t}=v_{c} ; \frac{d \varphi_{h}}{d t}=\omega_{h} ; \\
\frac{d v_{c}}{d t}=\frac{T_{c}-r_{c} F_{c}}{J_{c}} ; \frac{d v_{h}}{d t}=\frac{T_{h}-r_{h} F_{h}}{J_{h}} ; \\
\frac{d v_{c}}{d t}=\frac{\left(\begin{array}{l}
\left.F_{c}+m_{h} \omega_{h}^{2}\left(s_{h}-l_{h} / 2\right)+\left(m_{b}+m_{r}\right) \omega_{h}^{2} s_{h}-\right) \\
-F_{c l}-F_{h} \cos \beta-v_{c}\left(d m_{r} / d t\right)
\end{array}\right)}{m_{h}+m_{b}+m_{r}}=\frac{F_{c}-F_{\Sigma c l}}{m_{\Sigma}} ; \\
\frac{d \omega_{h}}{d t}=\frac{\left(\begin{array}{l}
F_{h} s_{h} \sin \beta-T_{h l}-2 m_{h} \omega_{h} v_{c}\left(s_{h}-l_{h} / 2\right)- \\
-2\left(m_{b}+m_{r}\right) \omega_{h} v_{c} s_{h}-\omega_{h} s_{h}^{2}\left(d m_{r} / d t\right)
\end{array}\right)}{m_{h}\left(s_{h}-l_{h} / 2\right)^{2}+m_{h} l_{h}^{2} / 12+\left(m_{b}+m_{r}\right) s_{h}^{2}}=\frac{F_{h} s_{h} \sin \beta-T_{\Sigma h l}}{J_{\Sigma}},
\end{array}\right.
$$

where the spring forces are described by:

$$
\begin{aligned}
& F_{c}=c_{c}\left(r_{c} \theta_{c}-\left(s_{h}-s_{h i}\right)\right) \\
& \left\{\begin{array}{l}
F_{h}=c_{h}\left(r_{h} \theta_{h}-\left(l_{b i}-l_{b}\right)\right), \text { if } l_{b}>l_{b i}-r_{h} \theta_{h} \\
F_{h}=0, \text { if } l_{b} \leq l_{b i}-r_{h} \theta_{h},
\end{array}\right.
\end{aligned}
$$

where $l_{b i}-$ the initial hoist cable length; $s_{h i}-$ the initial dipper handle stroke, and the variable parameters are computed as:

$$
\begin{gathered}
l_{b}=\sqrt{s_{h}^{2}+d_{b}^{2}-2 s_{h} d_{b} \cos \left(\alpha-\varphi_{h}\right)} \\
c_{h}=\frac{E S}{l_{b}+l_{d}}
\end{gathered}
$$

where $E$ - the tensile modulus; $S$ - the total cross section of the hoist cable.

The external loads in (1) are also variable parameters, where the forces $F_{c l}$ and $T_{h l}$ are random and gravity force is deterministic:

$$
\begin{gathered}
F_{c l}=F_{n}+g\left(m_{h}+m_{b}+m_{r}\right) \sin \varphi_{h} ; \\
M_{h l}=F_{t} s_{h}+g m_{h} \cos \varphi_{h}\left(s_{h}-l_{h} / 2\right)+g\left(m_{b}+m_{r}\right) \cos \varphi_{h} s_{h} .
\end{gathered}
$$

Despite the description, (1)-(4) applies only to electric rope shovels, the same ideology of obtaining the mathematical description is also valid for the draglines.

\section{Control algorithms}

In the synthesis of the control algorithms, the authors believe that the modern electric drive like the induction motor drive with the direct torque control is able to provide such performance of the motor as to equate the electromechanical part of the mechatronic system 
to the inertia-free source of electromagnetic torque. Then, the internal is a control loop of the spring forces, and the outer is a control loop of the bucket speed. Each control loop is equipped with a controller. As shown in Fig. 1, each spring force controller should calculate the required motor torque. The analytical design method of aggregated regulators allows us to synthesize the desired control algorithms for spring force controllers. This method allows providing the aperiodic transients with a given rate of response for interconnected nonlinear systems with a complex structure.

Let us consider in more detail the synthesis of the control algorithm of the crowd spring force. The following invariant manifold provides the implementation of the criteria of the spring force response:

$$
\Psi_{1 c}=F_{c}-F_{c}^{*}(t)=0,
$$

and the following functional equation ensures aperiodic transients:

$$
T_{1 c} \dot{\Psi}_{1 c}+\Psi_{1 c}=0
$$

where $T_{1 c}$ - the time constant, which determines the rate of approach to the invariant manifold $\Psi_{1 c}$.

Then, solving (6) relative $\Psi_{1 c}$ and using (1), the following equation can be obtained:

$$
T_{1 c} c_{c}\left(r_{c} v_{c}-v_{c}\right)-T_{1 c} \frac{d F_{c}^{*}}{d t}+F_{c}-F_{c}^{*}=0 .
$$

To link the equation (7) and the reference crowd motor torque $T_{c}{ }^{*}$, it is necessary to use (7) to form a new invariant manifold in such a variable as directly dependent on the $T_{c}$ according to (1):

$$
\Psi_{2 c}=v_{c}-\frac{1}{r_{c}}\left(\frac{1}{T_{1 c} c_{c}}\left(F_{c}^{*}-F_{c}+T_{1 c} \frac{d F_{c}^{*}}{d t}\right)+v_{c}\right)=0,
$$

and it is necessary to solve the new functional equation in analogy to (6):

$$
T_{2 c} \dot{\Psi}_{2 c}+\Psi_{2 c}=0
$$

where $T_{2 c}$ - the time constant, which determines the rate of approach to the invariant manifold $\Psi_{2 c}$.

By completing these calculations, the required control algorithm can be synthesized:

$$
\begin{gathered}
T_{c}^{*}=\frac{J_{c}}{r_{c}} \frac{d v_{c}}{d t}+\left(r_{c}-\frac{J_{c}}{T_{1 c} T_{2 c} c_{c} r_{c}}\right) F_{c}-\frac{J_{c}}{r_{c}}\left(\frac{1}{T_{1 c}}+\frac{1}{T_{2 c}}\right)\left(r_{c} v_{c}-v_{c}\right)+ \\
+\frac{J_{c}}{c_{c} r_{c}}\left(\frac{d^{2} F_{c}^{*}}{d t^{2}}+\left(\frac{1}{T_{1 c}}+\frac{1}{T_{2 c}}\right) \frac{d F_{c}^{*}}{d t}+\frac{F_{c}^{*}}{T_{1 c} T_{2 c}}\right) .
\end{gathered}
$$

Similarly, the control algorithm of the hoist spring force can be synthesized: 


$$
\begin{aligned}
T_{h}^{*}=-\frac{J_{h}}{r_{h}} & \left.\frac{d^{2} l_{b}}{d t^{2}}+\left(\frac{1}{T_{1 h}}+\frac{1}{T_{2 h}}\right) \frac{d l_{b}}{d t}\right)+\left(r_{h}-\frac{J_{h}}{T_{1 h} T_{2 h} c_{h} r_{h}}\right) F_{h}-J_{h} v_{h}\left(\frac{1}{T_{1 c}}+\frac{1}{T_{2 c}}\right)+ \\
& +\frac{J_{h}}{c_{h} r_{h}}\left(\frac{d^{2} F_{h}^{*}}{d t^{2}}+\left(\frac{1}{T_{1 h}}+\frac{1}{T_{2 h}}\right) \frac{d F_{h}^{*}}{d t}+\frac{1}{T_{1 h} T_{2 h}} F_{h}^{*}\right) .
\end{aligned}
$$

In accordance with Fig. 1, the spring force controllers provide the operation of the external speed control loop, which in turn determines the performance of the excavator. To provide the required performance of the rope shovels, it is necessary to control the speed of the dipper handle about the saddle block and the crowd speed by the hoist drive and the crowd drive with a reasonable accuracy. At the same time, the control system should generally provide the reduction of the dynamic loads. To solve both of the tasks the fuzzy logic controller or the adaptive controller can be applied.

These speed controllers allow the deviation of the instantaneous values of the bucket speed and the handle speed on the reference values within the limits defined by the random component of the load (it is usually no more than $\pm 5 \%$ ). However, the controllers and support the average rope shovel performance and minimize the dynamic component of the spring forces transients in the transmission, primarily in the ropes. These properties are fully consistent with the main idea of the proposed intelligent mechatronic system.

\section{Observer}

The above-mentioned controllers according to (1) require information about mechanical values, but some of them are immeasurable because of rope shovel construction. To estimate the immeasurable values the authors propose the observer.

The estimation basis on the drum angle positions $\theta_{c}$ and $\theta_{h}$ that are measurable and the motor electromagnetic torques $T_{c}$ and $T_{h}$ that are given by frequency converters [17] or obtaining from the special diagnostic computer system [35]. The estimated variables are the spring force $F_{c}$ и $F_{h}$, the crowd speed $v_{c}$, the speed of the dipper handle about the saddle block $\omega_{h}$ and the hoist cable length from the boom point sheaves to the bucket $l_{b}$. Besides, the observer estimates the total crowd drive load force $F_{\Sigma / c}$ and the total hoist drive load torque $T_{\Sigma l h}$. The estimated values are calculated from (1) by means of the inverse model method. The structure of the observer is shown in Fig. 3.

The practical realization of this observer is called the numerical differencing. This operation can lead to increasing of high frequency noises from the measuring system. To avoid potential estimation errors and provide high quality of controlling to relay on estimated values, the authors suggest using the numerical filter-differentiator with finite pulse response [14] according to the methodology [15]. 


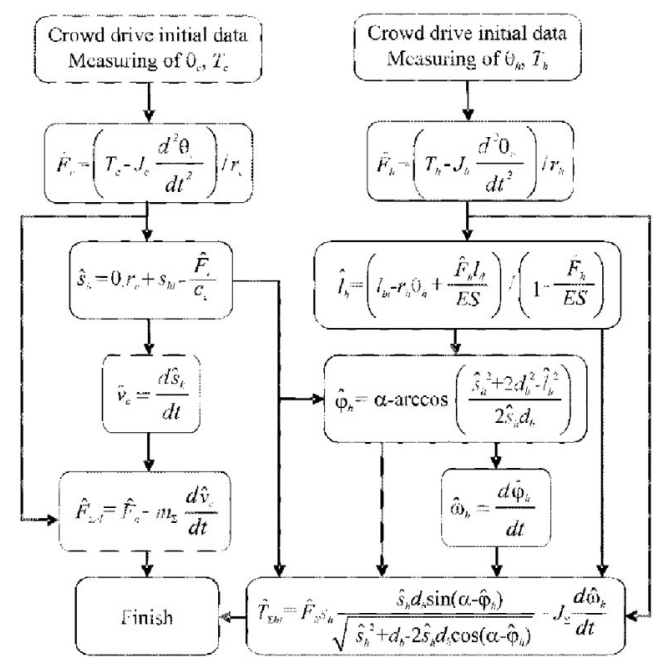

Fig. 3. The observer structure

\section{Experiments}

To prove the effectiveness of proposed development the authors have carried out two kinds of experiments. The first kind is experiments on location which have been carried out on the acting electric rope shovels. It confirms the adequacy of rope shovel model (1). The second kind is computational experiments. They help to investigate the effectiveness of the intelligent mechatronic system.

\subsection{Experiments on location}

The object of experiments on location was the rope shovel EKG-10 with mobile measuring system. This system provides the synchronous measuring of the drive currents and the angle positions of the crowd and hoist drums, the saddle block and the head block. Mounting of sensors was made with magnets and data exchange was organized with radio channel. The sensors location points are shown in Fig. 4. The processing of data was carried out with both the microcontroller and the laptop by means of a special program.

The rope shovel where the experiment was carried out was equipped with DC motor that's why to obtain the drive torques $T_{c}$ and $T_{h}$ it was necessary to measure the drive currents. The current sensors LT 1000-SI were used to measure current. The current data were transmitted to the measurement station where the data were analog to digital converted and then sent to the microcontroller of basic station. The angle position was got by the sensors based on two accelerometers. The sensors also included microcontroller ATmega-48 which was used for sending angle position data to the basic station. 


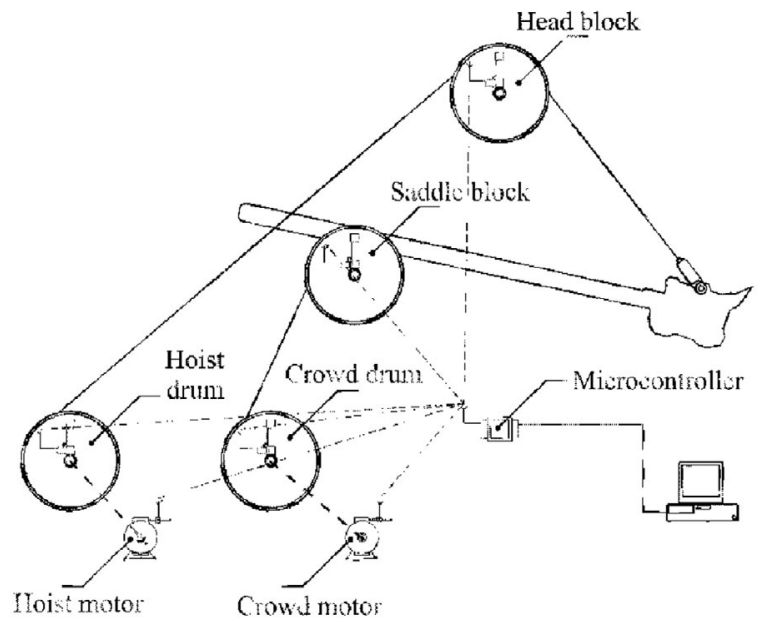

Fig. 4. The sensors location points

All above-mentioned data were read with laptop that realized the model (1) and at the same time calculated the speed of EKG-10 revolving elements from their angle positions as well as the spring forces. The comparison of these values from the model and from the direct calculation has confirmed the adequacy of (1). The maximum deviation was in the crowd speed at the start of digging and amounted to $25 \%$. The average deviation was $6.4 \%$ for the crowd speed, $3.4 \%$ for the speed of the dipper handle about the saddle block, $6.1 \%$ for the crowd spring force, and $8.8 \%$ for the hoist spring force.

\subsection{Computational experiments}

The result of the experiments on location allowed moving to the computational experiments. The object of these experiments was the same rope shovel EKG-10. The experiments were carried out with computer simulation of (1) by means of the self-made program. The parameters of EKG-10 model are presented in Table 1. The parameters of control algorithms (10) and (11) were selected based on the drive overload capacity of EKG-10. Thus the time constants $T_{1 c}, T_{2 c}, T_{1 h}$ и $T_{2 h}$ were set on values from $0.005 \mathrm{~s}$ to $0.05 \mathrm{~s}$. The observer contained the filter-differentiator with finite pulse response that had the cutoff frequency $4 \mathrm{~Hz}$ and the window to weigh with 500 counting breadth.

Initially the authors carried out the investigation of the spring force control quality with, the first, independent and, the second, corresponding operation of each drive. The independent operation result has shown in the maximum reference spring force the transient time is $0.27 \mathrm{~s}$ for the crowd drive and $0.23 \mathrm{~s}$ for the hoist drive. On the whole range of reference spring force the control error did not exceed $2 \%$ for the crowd drive and $3.5 \%$ for the hoist drive. The average observer estimation errors for all the values was $1.5 .3 \%$ and the maximum error didn't exceed 10\%. This observer accuracy has been sufficient condition for the controllers operation. The corresponding operation result has shown the spring force control quality almost did not depend on the total load of the both drive equally. It has confirmed the drive interconnections don't influence the spring force controllers operation mode and the accuracy of spring forces control. 
Table 1. The parameters of EKG-10 mechanical subsystem

\begin{tabular}{|c|c|c|}
\hline Parameter & Hoist drive & Crowd drive \\
\hline Transmission coefficient & 24 & 41 \\
\hline Drum radii, $\mathrm{m}$ & 0,64 & 0,45 \\
\hline Rope specific rigidity rate, $\mathrm{N} / \mathrm{m}$ & -* & $10,5.105$ \\
\hline Total specific inertia, $\mathrm{kg} \cdot \mathrm{m} 2$ & $10,3.103$ & $17,5.103$ \\
\hline Nominal bucket speed, $\mathrm{m} / \mathrm{s}$ & 1,1 & 0,81 \\
\hline Maximum rope force, $\mathrm{kN}$ & 1274 & 588 \\
\hline Boom angle to the horizon, rad & \multicolumn{2}{|c|}{0,79} \\
\hline Boom length from the saddle block to the head block, $m$ & \multicolumn{2}{|c|}{8,32} \\
\hline Dipper handle length, $\mathrm{m}$ & \multicolumn{2}{|c|}{11,37} \\
\hline Dipper handle mass, $\mathrm{kg}$ & \multicolumn{2}{|c|}{15.103} \\
\hline Bucket mass, kg & \multicolumn{2}{|c|}{$17,2.103$} \\
\hline
\end{tabular}

Finally, the circle of digging was simulated. In the simulation the trajectory of bucket moving was determined by the experiment on location. The simulation result has borne out the high quality of the spring force controllers operation. Comparison of the simulated spring force of each drive and the experimental one (Fig. 5) has noted the simulated values changed only because of operation mode technological requirements and they didn't carry additional oscillations.
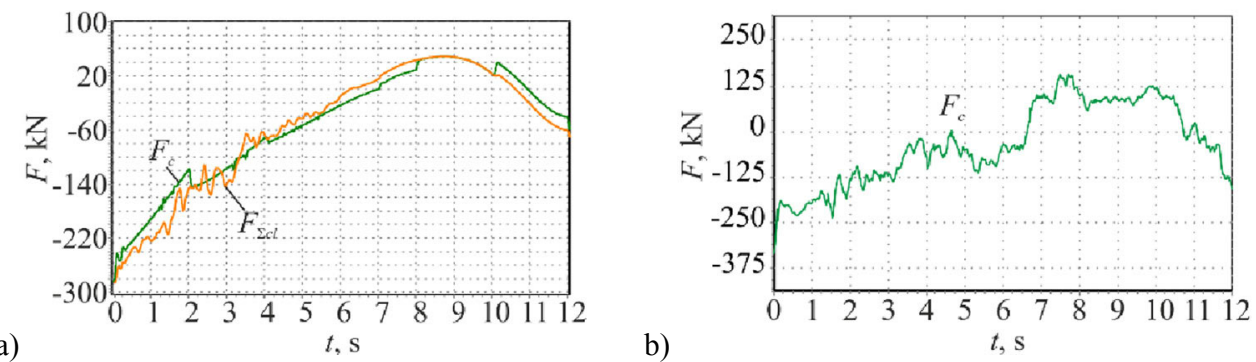

Fig. 5. Crowd rope spring force: a) simulation, b) experiment

Using the comparative criteria of dynamic loads decreasing that is based on amplitude spectrum analysis, the authors have found out that the dynamic load of crowd rope decreased by $19 \%$ and for the hoist rope the decreasing was $31 \%$.

Thus, the proposed intelligent mechatronic system for rope shovels ensures the high quality of controlling the dynamic state of the mechanical elements that avert drive overloads and high dynamic loads in the rope. It means this system performs all of prescribed criteria.

\section{Conclusions}

Notwithstanding rope shovels are the complicated robotic system, the intelligent mechatronic system based on mechanical subsystem nonlinear model decreases the dynamic loads in the transmission. The same ideology can also be successfully implemented for the Draglines. Realization of above-mentioned system requires the specific control algorithms, the estimation of the mechanical values and the new kind of 
measurement system. However, all these novelties can be embodied with electric drive equipment that is applied in the novel excavators.

The authors thank the staff of open pit mine "Mezhdurechye" and open pit mine "Vakhrushevsky" for the given opportunity to carry out the experiments on location.

\section{References}

1. K. Uno, K. Imaie, K. Maekawa, G. Smith, A. Suyama, Hitachi Review, 62, 99 (2013)

2. Graham Lumley, Matt McKee, Mining for efficiency (in PwC Australia, 2014)

3. Reliably Safely Sustainably Electrical Solutions For Mining (GE Energy, 2012)

4. S.E. Baybakov, Questions of electronics, 4:2, 112 (2012)

5. A.A. Artemyev, V.S. Potapenko, S.L. Ivanov, E.A. Kremcheev et al., Mining Equipment and Electromechanics, 9, 31 (2007)

6. CRCmining annual report (2004-2005)

7. Aditya Tirta Pratama, Armand Omar Moeis, Farizal., Proceeding of International Seminar on Industrial Engineering and Management, 7 (2011)

8. Enhancing efficiency in mining Safe and reliable control of motor-driven applications (ABB, 2014)

9. V.M. Zavyalov, I.Yu. Semykina, Proceedings of symposium "Advances in geotechnical and structural engineering”, Qingdao, China, 445 (2008)

10. S.V. Doronin, Proceedings of the universities. Mining Journal, 5, 86 (2007)

11. D.A. Kaminskaya, Proceedings of the universities. Mining Journal, 5, 154 (1975)

12. V.I. Kluchev, L.V. Zhiltsov, Yu.T. Kalashnikov, Electricity, 7, 28 (1981)

13. A.A. Bronov, V.P. Kochetkov, A.A. Kolesnikov, R.G. Reyfman, Proceedings of the universities. Mining Journal, 7, 106 (2007)

14. V.M. Zavyalov, I.Yu. Semykina, International transaction and application "Electric technic, electromechanics and electric technology", 69 (2007)

15. V. Kashirskikh, A. Gargayev, V. Zavyalov, I. Semykina, Proceedings of the 8th Russian-Chinese Symposium "Coal in the 21st Century: Mining, Processing, Safety», 274 (2016) 